\title{
HAVE YOU FELT BLUE OR RED? A COLOUR-BASED TOOL FOR CULTURALLY PLURAL TEAMS
}

\author{
Francesca MATTIOLI ${ }^{1}$ and Ingrid CALVO IVANOVIC ${ }^{1,2}$ \\ ${ }^{1}$ Politecnico di Milano, Italy \\ ${ }^{2}$ Departamento de Diseño, Universidad de Chile, Chile
}

\begin{abstract}
The extensive practice of collaborative design-based learning and the increasing cultural plurality of design courses in Design Education is becoming relevant. Students' critical reflection on teamwork experience through structured activities of self-, peer and group evaluation can provide a space for students to ask themselves pertinent questions and to discuss together with the team about each one's reflections. A guided sharing of personal perspectives on teamwork reveals to students the existence of multiple perceptions and increases mutual understanding and sensitivity. To this extent, a colour-based assessment tool for teams, the Teamwork Colour Matrix (TCM), was designed with the idea that colours can take on very different associations for each individual and therefore can leave more freedom of expression, especially when it comes to evaluating teamwork. Students intuitively coded their experience concerning the different moments of teamwork and represented it inside the TCM by using eight given colours. The paper presents the qualitative data collected during the TCM redesign and remote application, showing how it can support students to reflect and evaluate plural remote teamwork, at the same time it helps teachers to monitor team dynamics within collaborative design-based learning courses. The test also provided rich data about the challenges students experienced in the current collaborative distance learning context, contributing to acknowledging students' learning trajectories in this type of course.
\end{abstract}

Keywords: Culturally plural teams, design-based learning, visual communication, peer evaluation, self-evaluation

\section{INTRODUCTION}

The collaborative design-based learning approach is widely adopted to teach design in higher education institutions; according to it, students learn design by doing it collaboratively with other peers. These collaborative learning-by-doing practices are continuously evolving according to the socio-cultural changes around and within the educational context where they happen. In this regard, our interest specifically concerns the increasing cultural plurality of classes that have been determined by several contemporary factors such as the growing global mobility, the centrality of internalisation strategies in higher education and the emergence of interdisciplinary curricula, just to mention a few. These generalised phenomena are particularly significant in design education when students learn how to design through group projects. Therefore, collaborative learning becomes the stage where they can also improve their soft skills. Specifically, as described by the EU commission, global competencies are the ability to relate with culturally plural others being sensitive towards individual differences. [1] In recent years, the authors focused on the effective teaching strategies that can support the development of these skills in plural design education contexts. Particular attention was dedicated to self-, peer and group assessment [2] by designing structured moments and activities to promote students' reflective practice on soft skills [3]. The researchers glimpsed the possibility of conveying the students' reflection by using a shared language among all designers: visual communication, which is the basis of the design discipline. The use of the principles of visual communication is proposed, mainly through colour and shape, to activate the dialogue inside the team. The potentialities of visual language to communicate between cultures, generations, or plural groups of people are related to the human ability to deduct information and associate feelings and emotions to images or visual elements [4]. In the context of the Master of Science in Design \& Engineering at Politecnico di Milano, we developed a visual communication tool, the Teamwork Colour Matrix (TCM) [5], to support assessment and reflection on teamwork in plural teams where students represent the process of collaboration through an abstract 
colour composition. The preliminary test of the TCM in presence was very positive since the visual communication strategy turned out to be an effective and more culture-sensitive way to evaluate a process and people participating in it [5]. However, before we could further test the cardboard TCM version, design education faced another radical shift during the last year toward distance learning due to the pandemic emergency. This situation affected the in-class interactions among students and teachers and the student-to-student interaction within the team. Thus, the distance added a further level of complexity for teams, possibly aggravated by the stress conditions experienced by learners related to the pandemic and working from different time zones. Besides, teachers did not have in-class moments to observe students, adding a further obstacle to monitoring team dynamics. For all these reasons, it became urgent in our context to provide a virtual tool for students to evaluate teamwork and for teachers to monitor teams. Therefore, the TCM tool was redesigned for distant learning by adopting an action research approach and tested within a dedicated path on teamwork within the Master program named above. The paper presents the qualitative data collected during the tool testing. Eventually, the test provided rich data about the recurring difficulties students experienced in this particular education context, contributing to acknowledging the students' challenges in culturally-plural teams and those introduced by distant collaborative learning in traditional design courses.

\section{METHOD}

\subsection{Teamwork Colour Matrix: tool, sample, and remote application}

The TCM consists of a blank orthogonal grid designed to be the canvas that each student will fill with one or more colours, taken from a predefined 8-colours palette. Within the TCM, the horizontal axis represents a timeline divided into ten parts, where the left represents the beginning of collaboration activities while the right represents the end. These ten parts can be interpreted as single moments, summed portions, or time percentages during the collective experience of teamwork (fig. 1, teamwork timeline). Students are asked to evaluate teamwork by describing through colours the different moments lived and perceived, by associating one of the eight colours spontaneously and visually representing each part of the process. The diversity of the chromatic palette is intended to motivate the students to visually represent different moods, moments or meanings when communicating the teamwork experience [6]. In other words, the eight selected hues are conducive to be linked to positive-negative, intense-dull, active-passive, and simple-complex associations, among others. At the end of the activity, both group and individual evaluations are expressed inside the TCM as visual communication, in the shape of intuitive colour composition.
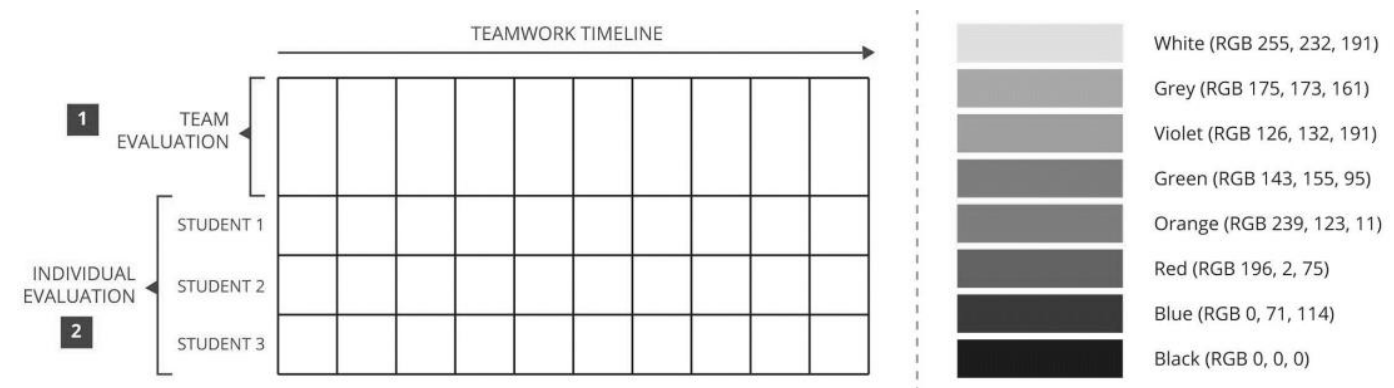

Figure 1. Teamwork Colour Matrix and the colour palette selected

The following instructions are provided to the students to do the exercise and thus create their own TCM about the teamwork they are experiencing:

1. Evaluate the team. In the first row (fig.1, Team Evaluation), the student has to evaluate the group and its dynamic by colouring each rectangle with one of the given colours. After colour selection, she is asked to produce a brief written description of the visual representation.

2. Evaluate self and peers. Each student evaluates individuals -the self and peers- by colouring the rectangles of the rows below the first (fig. 1, Individual Evaluation). Each row, made of 10 squares, is aimed at representing one team member. Again, the author writes detailed notes about the chromatic composition.

3. Present the TCM to teammates. Next, the team gathers, and each student shares her TCM. After individual presentations, the team should discuss possible issues emerging from team members and find solutions to improve teamwork. 
The cardboard version of the TCM was tested with 15 international students, during 2019, after the design studio of an interdisciplinary one-year master course. On that occasion, the TCM was physically created in the classroom, with cardboard and coloured paper. The main results showed that all students could evaluate teamwork by using visual communication through colours and that students relied very much on their TCMs to tell others their own experience within the team. An in-depth analysis of the first testing, explaining how the grid was designed, how the colour palette was selected, and the test results can be found in a previous paper published by the authors [5]. Due to the COVID-19 contingency, the TCM was adapted to a digital format. We applied the online version of the tool to 123 students of the MSc in Design \& Engineering of Politecnico di Milano. For the aim of this paper, the sample analysed consists of 32 students of the Product Development Design Studio I from very different origins (mainly Europe, Asia, and Latin America) and backgrounds (Design, Engineering, Robotics, Arts, Multimedia, Materials Science, among others).

The course was divided into nine cross-cultural teams of 3-4 people. Teams were decided by the students, and they were required to mix different origins and backgrounds inside the group. They worked together for four months and were supervised by three teachers, which are not part of the research team of this study. Within the course, a specific path was dedicated to teamwork called "Seminar on Teamwork" and led by the researchers as facilitators outside the teaching staff. As in the local education system teachers and learners have a strong hierarchy, teachers decided not to directly interfere with teams' dynamics nor push students with different sensitivity to open up in front of the professors. Therefore, the activities proposed during the Seminar were presented by the researchers outside of class time and were done autonomously by students. The seminar included three main activities for teambuilding and teamwork, namely 1) icebreaker and team formation, 2) team agreement and 3) self-, peer and group assessment after the first delivery. The TCM was done as part of the latter activity. The TCM was adapted to a PowerPoint presentation with 2 slides: the first presents the TCM blank grid and the colour palette available to the students. On this slide, students can perform the group, self, and peer evaluations, as indicated above, and provide the identification data for themselves and each of the teammates. In the second slide students can write their reflection on teamwork and each team member's performance. The activity started with a brief video explanation by the researchers about the matrix, the materials, and the exercise's goal, to proceed to individual work in both slides of the TCM. After the slides were completed, students were asked to gather in teams by using a classroom software to explain and discuss their results as a starting point to motivate conversation supported by visual communication. After the team discussion, students sent back their TCMs to the researchers for data analysis.

\subsection{Analysis strategy for the data obtained}

As the researchers did not know the students previously, the qualitative interpretation of data was based on visual communication and then verified with the written descriptions in the TCMs, without any additional information. The analysis focused mainly on three lines of study: a) to further validate visual communication as an effective strategy for plural students to share their feelings about the teamwork experience; b) to map recurring challenges that students experience in culturally-plural remote teams, and c) to provide guidelines for reading the visual elements inside the TCMs. These latter guidelines could support teachers in visually reading TCMs, without asking students to deliver explicit reports of their collaborative dynamics. Indeed, sharing their thoughts with educators could be perceived as particularly invasive according to individual cultures and sensitivity. To this extent, the researchers performed a visual analysis of group representation in the TCMs by focusing on the appearance of alignments/misalignments, colour uniformity/changes and the division of the TCMs into colour phases or moments inside the teamwork process. Then, we analysed the same visual elements regarding the individual performances by tracking colour analogies and differences between the author's colour representation of the self and the teammates and between each person's performance and teamwork representation. A cross-analysis between the group TCMs was then performed to understand the situations that emerged from a complete perspective. The visual analysis led to an interpretation of the process of teamwork, which was finally validated by reading the students descriptions of their TCM.

\section{RESULTS}

\subsection{Team 3: an example of data analysis and results}


Before proceeding with an account of the results obtained, an example of the analysis done is presented. In fig. 2, the TCMs created by the four team members (called here as M, R, J and F) are displayed, while in table 1, the qualitative observations noted by the researchers are reported together with the interpretation developed by cross-analysing the TCMs of the entire team.
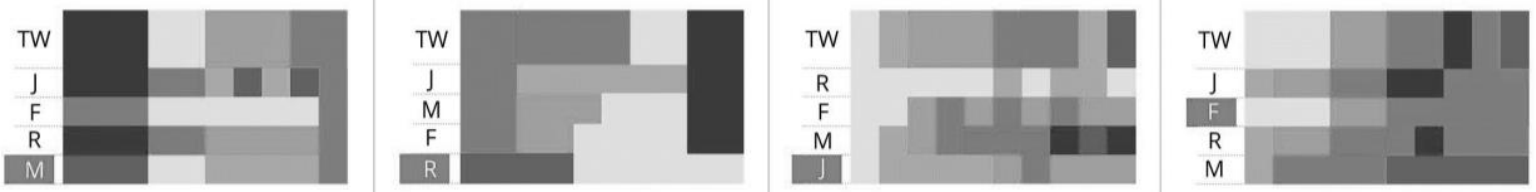

Figure 2. Example: TCMs of team 3, respectively made by students $M, R$, $J$ and $F$

The final row of the table is dedicated to a summary of the descriptions written by students, used to corroborate the interpretation. It is possible to notice that the interpretation matches the inner team dynamics without details about the specific facts.

Table 1. Example: TCMs made by team 3, respectively made by students $M, R$, $J$ and $F$

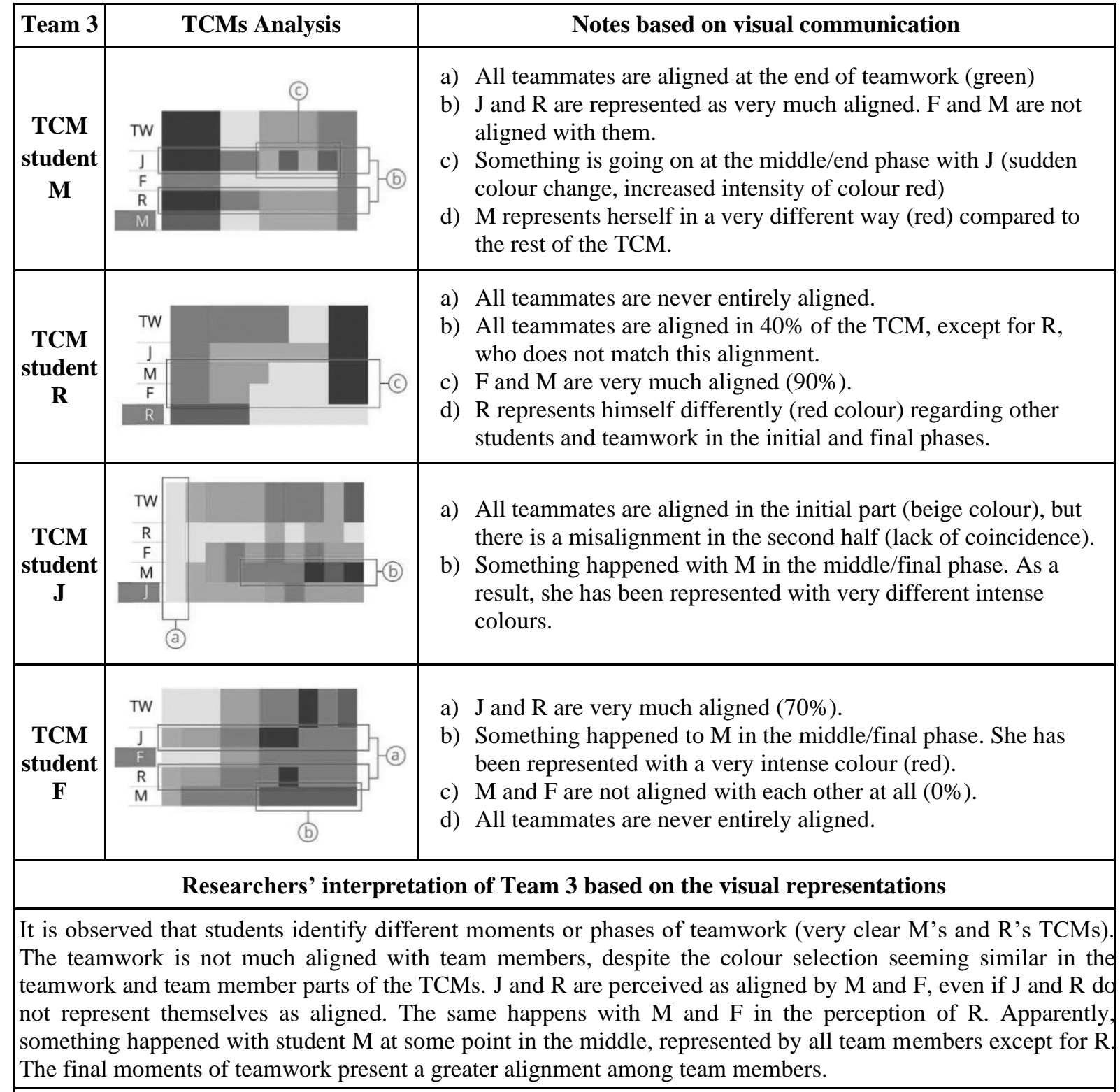

\section{Summary of students' descriptions}

All team members describe teamwork very positively, except, for $\mathrm{J}$ who acknowledges a conflict at the middle/end phase. In the beginning, team members did not know each other, and everybody describes the initial moment as a misaligned one, especially $\mathrm{F}$, who initially was in her hometown and described distance as an obstacle for teamwork. The final phase was stressful, but all the team was focused on the delivery. 
From J's perspective, a division in the team exists: the two girls are designers ( $\mathrm{M}$ and $\mathrm{F}$ ) on one side, and the two boys are engineers ( $\mathrm{R}$ and $\mathrm{J}$ ) on the other. Both $\mathrm{M}$ and $\mathrm{J}$ reported moments of discussion in the middle/final part of teamwork. Student J mentions that the "design couple" excluded the "engineers" because they were not skilled enough in using a specific design software. In his words, there was an explicit call for peer learning addressed to the designers, also for R, who mentions the talent of the two designers and his desire to learn from them. The others' storytelling supports the idea that the two boys were very cohesive with each other, and the girls, despite some differences, were very much connected, aligned, and balanced.

\subsection{Results account}

The results obtained from the visual analysis that could provide some guidelines for reading the TCMs refer mainly to the appearance of analogies and differences among the chromatic representations. In group and individual performances, most students use the resources of colour similarity and changes to visually mark the duration, the beginning and end of the different moments or phases perceived in the teamwork process. This constancy or inconstancy in the application of colour visually informs the teacher about possible situations or conflicts in the group dynamics, which is reinforced when some coincidences can be seen between students' compositions belonging to the same group. Some of the essential visual elements to notice are:

- Alignments, similarities, and coincidences: within one specific TCM or even in the comparison between the TCMs of the group members, colour alignments are a valuable visual element to observe the students' perception of how the team worked. As the different individual performances can be compared with the group performance inside of a TCM, it is possible to observe which students were (or not) aligned with the teamwork (e.g., the alignments between a part of the students of the group or misalignments of one student with the rest of the group). Also, it is relevant to note the moments in which the entire group was aligned (or not) and at what stage of the teamwork process these coincidences occur. For instance, in most cases, teammates divided the teamwork process into a matching number of colours (3-4 moments or phases within the teamwork).

- Colour differences, sudden changes, and contrast: when noticeable differences or suddenly it appears a great contrast in the application of colour for the representation of teamwork or individual performances, it is usually an indicator of a particular situation or issue, a conflict between teammates, a moment of high stress (e.g., delivery dates) or a very different individual attitude from that of the rest of the group.

- High colour intensity vs achromaticity: although the students do not receive any reference on colour psychology before completing the TCM, the results show a great variety of semantic colour associations. Without going into the specificity of colour language, it is possible to notice the appearance of colour saturation -intense or vivid colours- to indicate intense positive or negative moments during teamwork (e.g., stressful events, discussion between teammates, a very productive phase or high enthusiasm about work). On the other hand, achromatic colours (grey and black) are usually used by students to represent a passive attitude to work, dull moments within the teamwork, confusion, lacking motivation or understanding between the team members.

Another significant result is that all the students involved in the test communicated as much visually as in words, providing a pretty accurate overview of their teamwork experiences. Regarding the tool, it is worth saying that the cross-analysis of the TCMs provided an accurate reading of each team and individual student contribution; this supports the idea that abstract visual communication could effectively enhance self-, peers and group assessment activities. The descriptions provided by each student were essential to understanding specific situations, behaviours or conditions that influenced teamwork dynamics. Another relevant result is that a straightforward plural attitude toward assessment emerged since not all the students demonstrate the same confidence in making explicit reference to the critical points of collaboration, such as conflicts and disagreements (e.g., J was the only one reporting a division in team 3). Still respecting individual and cultural differences in dealing with disputes, the TCM visual representation prompted each student to communicate alignments and misalignments with others and with the overall process of teamwork. This description is highly detailed regarding time and interrelations in the team, but it is generic on the specific facts or behaviours due to its abstraction.

From student's storytelling, some recurring elements emerged. The majority of students described teamwork by dividing the grid space into phases and reporting them in their descriptions. As external observers, the identification of these phases was the first step to analyse the TCMs. The main result is that all teams experienced three phases: an initial phase dedicated to knowing the others in the group; a middle phase where students report moments of misalignment due to various reasons (e.g., 
misunderstandings, lousy communication, different approaches to teamwork, decisions to take, lack of skills, bad revisions with professors) and, a very intense final phase before the delivery. It also emerges that the designers often tend to push aside engineers who do not already have some design-related skills within the course, which is highly design-oriented. In several cases (e.g., team 3), engineers make explicit calls for peer learning towards their design colleagues. Finally, students' descriptions highlight that, in this blended teamwork (i.e., some students at the university and some in their hometowns), those who worked remotely had trouble effectively contributing to collaboration and were sometimes excluded by the other team members.

\section{DISCUSSION}

The results show that all the students could assess the self, peers and group using visual communication, which guaranteed freedom in the evaluation itself. Students' abstract representation of the teamwork experience was fundamental for an external observer to gain an overall understanding of the process and contributions of each student. The method developed and described to interpret the TCMs was validated and demonstrated to be accurate and possibly replicable by other researchers or teachers. Indeed for 8 teams out of 9 , the interpretation based on the visual elements was coherent with the descriptions provided by students, similarly to what happens with team 3 . Some relevant themes and challenges arise from students' visual compositions and storytelling and are here briefly addressed. Firstly, the process of teamwork is often visually divided into phases which usually correspond to an initial phase of ice breaking, lack of mutual understanding or knowledge; then a middle phase where the team faces issues or challenges; and a final intense phase before the deadline for the delivery, which appears to be often stressful and related to arguing. In two teams, these arguments took place in an early phase, leading the teams to a deeper discussion and collective agreement on the collaboration rules; in these groups, the final phase appeared to be less stressful and conflictual. Another recurring topic is the division of the tasks within teams, often led by designers intended as the "experts" of the design process and sometimes resulting in the exclusion of engineers from several tasks related to concept development, visual research, and project display. This phenomenon was also detected when students, regardless of their background, demonstrate a lack of hard skills in some domains. Future research should envision ways to develop teaching activities to structure peer learning within teams, possibly reducing low-skilled students' exclusion and enhancing the learning experience for all. The last topic worth mentioning is the difficulties experienced by students working from a distance, who turned out to be often excluded or somehow disadvantaged in participating in team activities. Also, due to the historical moment we are facing, it is crucial to address this reality by structuring teaching and learning activity that can effectively promote the inclusion of distant students in blended learning. For all these reasons, the TCM test was very positive, and also, as a teaching activity, it supported the understanding of the new learning realities encountered by students, which in our view should be expanded and addressed by institutions and teachers. In this regard, the TCM constitutes the first step to raise awareness of the importance of teamwork as a learning experience in design-based courses and could hopefully be used by students to reflect on their learning as much as teachers monitor and support teams they supervise.

\section{REFERENCES}

[1] European Commission. Key competencies for lifelong learning, 2007, pp. 1-103, Brussels.

[2] Mattioli F. and Ferraris S. Self-evaluation and peer evaluation tool for design and engineering teams: experiment conducted in a design studio. In Towards a New Innovation Landscape: Proceedings of The 21st International Conference on Engineering and Product Design Education, 2019, pp. 364-369.

[3] Sluijsmans D., Dochy F. and Moerkerke G. Creating a learning environment by using self-, peerand co-assessment. Learning Environments Research, 1(3), 1999, 293-319.

[4] Dondis D. A. La sintaxis de la imagen. Barcelona: Gustavo Gili, 1976.

[5] Calvo Ivanovic I. and Mattioli F. (2021). A colour is worth a thousand words! A colour-based tool to foster communication in culturally-plural teams. In The International Scientific Conference of the Colour Society of Russia: Selected papers, 2021, pp. 125-130.

[6] Jonauskaite D., Althaus B., Dael N., Dan-Glauser E., and Mohr C. What colour do you feel? Colour choices are driven by mood. Colour Research \& Application, 44 (2), 2019, pp. 272-284. 